\title{
Dynamic changes of the extracellular matrix during corneal wound healing
}

\author{
Elvira Lorenzo-Martín a, Patricia Gallego-Muñoz ${ }^{\text {a }}$, Santiago Mar ${ }^{\text {b }}$, Itziar Fernández c, d, Pilar Cidad e, \\ M. Carmen Martínez-García a, * \\ a Departamento de Biología Celular, Histología y Farmacología, Facultad de Medicina, Grupo de Investigación Reconocido: Técnicas Ópticas para el Diagnóstico, Universidad de Valladolid, \\ Valladolid, Spain \\ b Departamento de Físico Teórica, Atómica y Óptica, Facultad de Ciencias, Grupo de Investigación Reconocido: Técnicas Ópticas para el Diagnóstico, Universidad de Valladolid, Valladolid, Spain \\ ${ }^{\text {c } C I B E R-B B N}$ (Centro de Investigación Biomédica en red en Bioingeniería, Biomateriales y Biomedicina), Spain \\ d Instituto de Oftalmobiología Aplicada, Universidad de Valladolid, Valladolid, Spain \\ e Departamento de Bioquímica, Biología Molecular y Fisiología, Universidad de Valladolid-Consejo Superior de Investigaciones Científicas, Valladolid, Spain
}

\section{A R T I C L E I N F O}

\section{Keywords:}

Extracellular matrix

Collagen type III

Lumican

Decorin

Corneal wound healing

Rabbit

\section{A B S T R A C T}

The extracellular matrix (ECM) confers transparency to the cornea because of the precise organization of collagen fibrils and a wide variety of proteoglycans. We monitored the corneal wound healing process after alkali burns in rabbits. We analyzed the location and expression of collagens and proteoglycans, the clinical impact, and the recovery of optical transparency.

After the animals received both general and ocular topical anesthesia, the central cornea of the left eye was burned by placing an $8-\mathrm{mm}$ diameter filter paper soaked in $0.5 \mathrm{~N} \mathrm{NaOH}$ for $60 \mathrm{~s}$. The eyes were evaluated under a surgical microscope at 1,3 , and 6 months after burning. At each time point, the clinical conditions of the burned and control corneas were observed. The arrangement of collagen fibers in the corneal stroma was visualized by Picrosirius-red staining, Gomori's silver impregnation and transmission electronic microscopy. Corneal light transmittance was also measured. Myofibroblasts presence was analyzed by immunohistochemistry. mRNA expression levels of collagen types I and III, lumican, decorin, keratocan and alpha-smooth muscle actin were determined by quantitative real-time polymerase chain reaction.

One month after alkali burn, the ECM was disorganized and filled with lacunae containing different types of cells and collagen type III fibers in the wound area. Corneal opacities were present with attendant loss of light transmittance. Collagen and proteoglycan mRNA expression levels were up-regulated. After three months, wound healing progress was indicated by reduced corneal opacity, increased light transmittance, reorganization of collagen fibers and only collagen type I expression levels were at control levels. After six months, the wound area ECM morphology was similar to controls, but transmittance values remained low, denoting incomplete restoration of the stromal architecture.

This multidisciplinary study of the stromal wound healing process revealed changes in corneal transmittance, collagen organization, myofibroblasts presence and ECM composition at 1, 3, and 6 months after alkali burning. Documenting wound resolution during the six-month period provided reliable information that can be used to test new therapies.

\begin{tabular}{|ll}
\hline Abbreviations \\
$\alpha$ SMA & Alpha-smooth muscle actin \\
ACTB & Actin beta \\
COL1A1 & Collagen type I alpha 1 \\
COL3A1 & Collagen type III alpha 1 \\
Ct & Threshold cycle \\
ECM & Extracellular matrix \\
KERA & Keratocan \\
\hline
\end{tabular}


eye, the cornea, is an avascular and highly innervated tissue characterized by a curved shape and transparency. The cornea is the major refractive component of the eye, and thus it must not impede the passage of light (Hatami-Marbini and Etebu, 2013). The cornea consists of three structures: two epithelial layers, outer and inner, and the stroma or middle layer. The stroma represents approximately $90 \%$ of the total corneal thickness and is composed of keratocytes and a collagen-rich extracellular matrix (ECM) that is organized into layers termed "lamellae". The keratocytes are the principal cell type that maintains the ECM. These cells, which are evenly located between the stromal lamellae, usually exist in a fairly quiescent state (Petroll and Miron-Mendoza, 2015; Singh et al., 2012; West-Mays and Dwivedi, 2006).

The transparency of the corneal stroma differs from other collagenous structures and results from the precise organization of the ECM (Boote et al., 2003; Maurice, 1970), which consists of mostly of collagen fibrils that interact with proteoglycans. The collagen fibrils are uniformly $20 \mathrm{~nm}$ in diameter and are organized into fibril bundles that are then organized into fibers. The collagen fibers form the stacked lamellae that are arranged orthogonally from each other (Chen et al., 2015; Radner et al., 1998).

Collagens are the most abundant proteins in all connective tissue, and, to date, 28 different types of collagens have been identified (Karsdal et al., 2017; Ricard-Blum, 2011). The major fibril-forming collagens of the corneal stroma are types I and V. Other minor collagen types like collagen III, IV, and VII are essential in other corneal structures like basement membranes and during ECM remodeling (Hassell and Birk, 2010; Massoudi et al., 2016). Corneal collagen type III is highly expressed in the healing cornea and, together with the loss of the organization of the collagen type I fibrils, plays an important role in scarring. Collagen type III is considered to be one of the principal markers of fibrosis (Galiacy et al., 2011; Massoudi et al., 2016).

In addition to collagens, there is a wide diversity of proteoglycans in the cornea that are associated with specific groups of collagen fibrils and play key roles in collagen fibrillogenesis, matrix assembly, and therefore in corneal transparency (Massoudi et al., 2016; Michelacci, 2003; Scott and Haigh, 1985). Keratan sulfates, such as lumican and keratocan, and dermatan sulfate proteoglycans such as decorin constitute the main proteoglycans in the corneal structure. All of them are small leucine-rich proteoglycans that interact with different regions of the collagen molecules, stabilizing the collagen fibrils once formed. They have key roles in the regulation of the growth and assembly of collagen fibrils (Hassell and Birk, 2010; Liu et al., 2003; Neame et al., 2000). In addition to binding collagen, they have a key role interacting with other molecules that regulate cell growth, apoptosis and inflammation (Chakravarti, 2006).

When the cornea is exposed to different types of damage, like surgery, physical trauma, or chemical burns, the wound healing begins but the process is different depending on the type of injury. Injuries occasioned by alkali and acid agents represent $11.5-22.1 \%$ of ocular traumas and are some of the most difficult to treat (Sharma et al., 2018). The recovery from these types of damage is a complex process that requires the replacement of injured cells and tissues (Leask and Abraham, 2004). During wound healing, the quiescent keratocytes present in the stroma are activated and transitioned into the repair phenotypes fibroblasts and myofibroblasts that synthesize and release ECM components in the wound area. Myofibroblasts proliferate and migrate towards the site of the injury to close the wound by depositing excessive amounts of ECM proteins, including collagens type I, III, IV, and V to facilitate tissue repair (Chaurasia et al., 2015; Gallego-Munoz et al., 2018; Tandon et al., 2010). During this process, the collagen type I/type III ratio can be disrupted, and this fact has been described as one of the main reasons for corneal opacity after corneal injury (Chen et al., 2005). Moreover, myofibroblasts produce large amounts of proteoglycans, including decorin and lumican, that play a key role stabilizing the disorganized ECM (Hassell and Birk, 2010; Massoudi et al., 2016).

When the wound healing process is completed, myofibroblasts usually disappear by apoptosis or transdifferentiation into keratocytes (Barbosa et al., 2010; Kaur et al., 2009). The transdifferentiation of myofibroblasts is a crucial feature of tissue repair (Klingberg et al., 2013), and the failure to undergo it has been identified in multiple pathologies (Gabbiani, 2003; Radisky et al., 2007; Saika et al., 2008; common during the wound healing process, but its persistence is considered a stromal complication of the repair process.

Several animal models of alkali burn have been used to evaluate the corneal wound healing process (Choi et al., 2017) and to test drugs that reduce the scarring or corneal opacity (Griffith GL et al., 2018; Haddox et al., 2001; Lopes et al., 2017; Zahir-Jouzdani F, 2018). All of the procedures use sodium hydroxide to produce the alkali burn. However, each model uses different animal species, solution concentrations, and time of alkali exposure, depending on the experimental objective and methods (slight or severe injuries, neovascularization or not, histological or molecular studies, etc.). Furthermore, most of the studies are short-term and do not take a multidisciplinary approach. In our study, we have used a standardized rabbit model of severe alkali burn injury (Chung et al., 1987; Gallego-Munoz et al., 2019) to make a multidisciplinary and long-term study.

We agree with Karsdal et al. (2017) regarding "the importance of having the right collagen at the right place and the right time" for proper wound healing. For this reason, our study seeks to connect the dynamic changes of ECM component expression, including collagens and other molecules, with the morphological demonstration of their localization. The molecular-level changes are then associated with the clinical impact and the recovery of optical transparency. Recognizing that wound healing is a long process, the study was conducted over periods of one, three, and six months.

\section{Materials and methods}

This study was approved by the Animal Research and Welfare Ethics Committee of the University of Valladolid (Spain) in agreement with European (Council Directive 2010/63/UE) and Spanish regulations (RD 53/2013). Animals were cared for following the guidelines of the Association for Research in Vision and Ophthalmology (ARVO) Statement for the Use of Animals in Ophthalmic and Vision Research. Female New Zealand albino rabbits, 12-15 weeks old, weighing 2.5-3 kg, were supplied by Granja San Francisco, Navarra, Spain, a center listed in the official Spanish Registry as a provider of lab animals.

\subsection{Alkali burn procedure}

Thirty-four New Zealand rabbits were anesthetized with a thigh intramuscular injection of ketamine hydrochloride $(37.5 \mathrm{mg} / \mathrm{kg}$, Ketolar, Parke Davis SA, Barcelona, Spain) and xylazine hydrochloride $(5 \mathrm{mg}$ / kg; Rompun, Bayer, Leverkusen, Germany), followed by topical application of $0.5 \%$ tetracaine hydrochloride and $1 \mathrm{mg}$ of oxybuprocaine (Colircusi Anestésico Doble, Alconcusí SA, Barcelona, Spain). A standardized corneal alkali (Gallego-Munoz et al., 2019) wound model was used. Briefly, corneal alkali burns were induced by placing an 8-mm diameter filter paper soaked in $0.5 \mathrm{~N} \mathrm{NaOH}$ solution $(\mathrm{pH}$ 14.7) on the central cornea of the left eye of each rabbit. After $60 \mathrm{~s}$, the filter paper was removed, and the eye thoroughly irrigated with sterile phosphate-buffered saline solution (PBS). The eyes of a similar number of control animals had all of the same steps as the experimental eyes but without the exposure to $\mathrm{NaOH}$.

\subsection{Clinical course}

The anterior segment of each eye was evaluated under surgical microscope (Leica M220 F12; Leica Microsystems, Nussloch, Germany) before burning and afterwards at each time point. Corneal opacity was graded following an adaptation of the Fantes' scale (Fantes et al., 1990): $0=$ no opacity; 1 = slight opacity with pupillary margin and iris details visible; $2=$ moderate stromal opacity with pupillary margin partially visible; $3=$ significant stromal opacity with pupillary margin not visible; and $4=$ intense stromal opacity with more whitish color (Choi et al., 2017; Gupta et al., 2011).

\subsection{Transmittance measurements}

Corneal transmittance was measured with a spectrometer that was 
illuminated the excised cornea that was secured in a corneal holder (described below), and the transmitted light was collected in a monochromator that incorporated an Optical Multichannel Analyzer (OMA; model 1460; 509 channels; EG\&G Princeton Applied Research) and a computer to reduce the measurement time as much as possible (details provided below). Rabbits were randomly assigned into 1-, 3-, and 6-month follow-up groups ( $n=5$ rabbits each) and euthanized by intracardiac injection of sodium pentobarbital (Dolethal ${ }^{\circledR}$ 0737-ESP; Vetoquinol, Madrid, Spain) under general anesthesia. The 10 corneas (5 experimental, 5 control) were removed at each time point. The corneas were placed between the two Teflon supports of the corneal holder, creating a closed chamber through the O-rings. The Teflon holder had a curvature similar to the cornea and included a 2-mm diameter hole with quartz optical windows that allowed light to pass. Sterile $\mathrm{NaCl}$ was passed through the cornea holder to maintain cleanliness, sample temperature, and pressure similar to intraocular pressure. Light was applied using different lamps with power sources stabilized according to the spectral range of measurement. The spectrometer consisted of a Jarrell-Ash monochromator of $300-\mathrm{mm}$ focal length equipped with a $1,200 \mathrm{l} / \mathrm{mm}$ diffraction grating with maximum efficiency in the visible range. The monochromator collected light in the spectral range of $400-750 \mathrm{~nm}$ and detected by the OMA. The combinations of the monochromator scan and the characteristics of the OMA allowed collection of spectral scans in 10-15 min with a resolution of $1 \mathrm{~nm}$. Spectral transmittances were measured in one sweep with the cornea in the corneal holder and again without a cornea but with the $\mathrm{NaCl}$ maintenance solution. The quotient of both measurements (burned/control) was taken as the spectral transmittance of the cornea.

\subsection{Tissue processing}

After transmittance measurements, the corneas were fixed with $4 \%$ buffered paraformaldehyde and embedded in paraffin. Sections $5-\mu \mathrm{m}$ thick were stained with Picrosirius-red $(0.1 \%$ Sirius red in saturated aqueous picric acid for $1 \mathrm{~h}$ ) (Junqueira et al., 1979). Sections were examined under an Axiophot microscope (Zeiss Axiophot HBO-50; Carl Zeiss, Oberkochen, Germany) with polarized light. Photomicrographs were captured using the AxioCam HRc Digital Camera and Axiovision release 4.8 software (Carl Zeiss) under the same plane of polarization. Polarization colors are associated with different collagen fiber thicknesses, packing density, and spatial arrangement (Dayan et al., 1989). Orange to red colors were correlated with thick collagen fibers that were tightly packed and well aligned. Yellowish-green colors were correlated with thinner fibers that were less tightly packed.

Other $5-\mu \mathrm{m}$ sections were stained with a modification of the Gomori's Silver Impregnation technique to demonstrate the presence of collagen type III fibers [54]. The sections were examined under an Axiophot microscope (Zeiss Axiophot HBO-50; Carl Zeiss, Oberkochen, Germany) and photomicrographs were obtained with an AxioCam HRc Digital Camera and Axiovision release 4.8 software (Carl Zeiss).

\subsection{Alpha-smooth muscle actin ( $\alpha S M A)$ immunohistochemistry}

Sections $5-\mu \mathrm{m}$ thick were deparaffinized and alpha-smooth muscle actin ( $\alpha \mathrm{SMA})$ presence, a marker of myofibroblasts, was identified by incubation with mouse monoclonal anti-human $\alpha$ SMA Clone $1 \mathrm{~A} 4$ Ready-to-use (Dako, Glostrup, Denmark). The secondary antibody was FITC goat anti-mouse IgG (Molecular Probes). Nuclei were stained with DAPI (Molecular Probes). Immunofluorescence sections were examined under an Axiophot fluorescence-incorporated microscope (Zeiss Axiophot HBO-50; Carl Zeiss, Oberkochen, Germany) and photomicrographs were captured using the AxioCam HRc Digital Camera and Axiovision release 4.8 software (Carl Zeiss). All the $\alpha$ SMA-positive cells were then counted at 100X magnification using the Touch Count function from Cell A software (Olympus) in $300,000 \mu \mathrm{m}^{2}$ areas of the wound and peripheral area. The percentages were determined relative to the total cell number at each time point.

All images were taken under the same conditions and experiments were performed at least four times. Limbal blood vessels were used as

\subsection{Transmission electron microscopy (TEM)}

Four corneas were taken for electron microcopy before burning (control) and 1, 3 and 6 months after burning. They were fixed in $2 \%$ glutaraldehyde and $2 \%$ paraformaldehyde and post-fixed in $1 \%$ osmium tetroxide. The fixed tissues were embedded in Spurr's plastic. Selected sections were cut on LKB ultramicrotome (Prodkter Ab, Stockholm Sweden) and examined under a Jeol JEM-1011HR electron microscope (Jeol Ltd., Tokyo, Japan) of the Microscope Unit of the Parque Científico-Universidad de Valladolid. Photomicrographs were obtained with the ES1000W Erlangshen CCD Camera (Gatan Inc., Pleasaston, USA).

2.7. RNA isolation, $C D N A$ synthesis, and quantitative real-time polymerase chain reaction

To estimate gene expression, another ten corneas ( 5 burned and 5 control) at each time point were excised and divided with an 8-mm trephine into wounded and peripheral areas. Total mRNA was isolated from each area with Trizol LS Reagent (Applied Biosystems [ABI], Life Technologies, Foster City, CA, USA) following the manufacturer's protocol. In three independent experiments, the mRNA was reverse-transcribed to cDNA, using the high-capacity cDNA reverse transcription kit with RNAse inhibitor [ABI], following the vendor's instructions. Quantitative real-time polymerase chain reaction (qRT-PCR) was conducted on a Rotor-Gene 300 instrument (Corbett Research UK, Ltd., Cambridge, UK) using the ABI TaqMan Gene Expression Master Mix and the ABI custom TaqMan gene expression assay [ABI]. The assayed genes were as follows: collagen type I alpha 1 (COL1A1; Oc03396073_g1), collagen type III alpha 1 (COL3A1; Oc04096544_s1), lumican (LUM; Oc03395736_s1), and decorin (DCN; Oc03398036_m1), keratocan (KERA; Oc03396149_m1), alpha-smooth muscle actin ( $\alpha$ SMA; Oc03399251_m1). The ABI TaqMan gene expression assay for actin beta (ACTB; Oc03824857_g1) was used as the housekeeper gene and control corneas as the calibrator. Reactions were run in triplicate in total volumes of $20 \mu \mathrm{L}$. Each gene expression level was determined at the different time points using the threshold cycle $(\mathrm{Ct})$ relative quantification method $\left(2^{-\Delta \Delta C t}\right)$ and represented as $\log \left(2^{-\Delta \Delta C t}\right)$.

\subsection{Statistical analysis}

Gene expressions were summarized as means \pm standard deviations (SD). The linear mixed-effect model was used to evaluate the effect of visit, area, and interaction on the expression of each gene. "Subject" was included as a by-subject random effect. "Visit" (1, 3, and 6 months) and "area" (wound vs. peripheral area) were included as categorical variables in each linear mixed-effect model. Wound area opacity was evaluated in an ordinal scale. Median and interquartile range were used to describe opacity in each visit. The global effect of visit was evaluated using the Friedman's non-parametric test. Pairwise comparisons were based on a permutation test of symmetry for paired ordinal data. The statistical analyses were performed using the $\mathrm{R}$ statistical package version 3.4.2 (R Core Team. Foundation for Statistical Computing, Vienna, Austria. URL: https://www.R-project.org/). P-values < 0.05 were considered statistically significant.

\section{Results}

\subsection{Clinical results}

Corneal opacity was present in burned corneas until the end of the study (Fig. 1A). The maximum grade was at 1 month, and then it decreased progressively. The opacity was significantly lower at 6 months than at 1 month after burning ( $\mathrm{p} \leq 0.01$, Fig. 1B).

\subsection{Transmittance}

The burned/control transmittance ratio was very low at 1 month and increased progressively until the end of the study, but without 

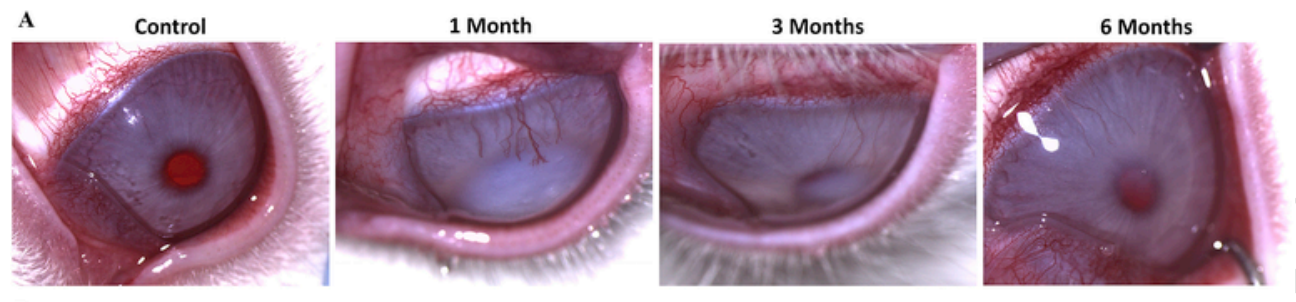

B

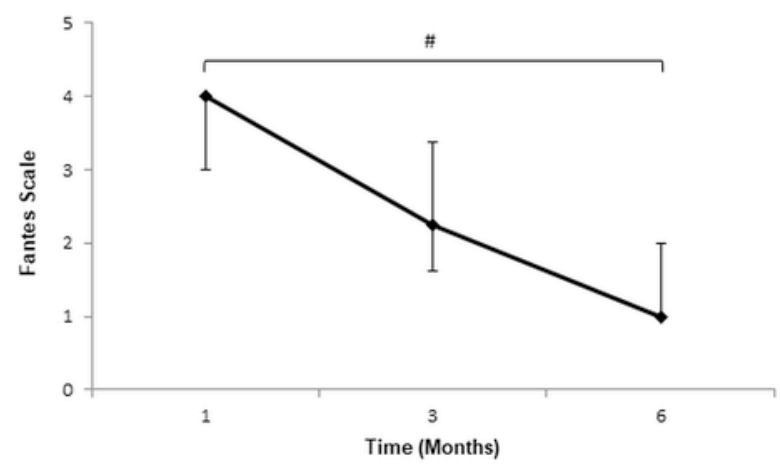

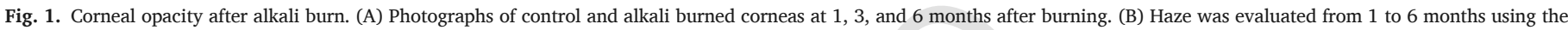
adaptation of Fantes' scale. The opacity grade was significantly lower at 6 months than at 1 month after burning (\#, p $\leq 0.01)$.

trol values (Fig. 2). Moreover, the transmittance ratio and restoration were dependent on the wavelength measured at each time point. The transmittance values at high wavelengths $(750 \mathrm{~nm})$ were greater than at lower wavelengths $(400 \mathrm{~nm})$.

\subsection{Histological assessment}

\subsubsection{Picrosirius-red and Gomori's silver impregnation staining}

In control corneas, Picrosirius-red staining revealed orange to red polarization colors in the anterior stroma, indicating the presence of tightly

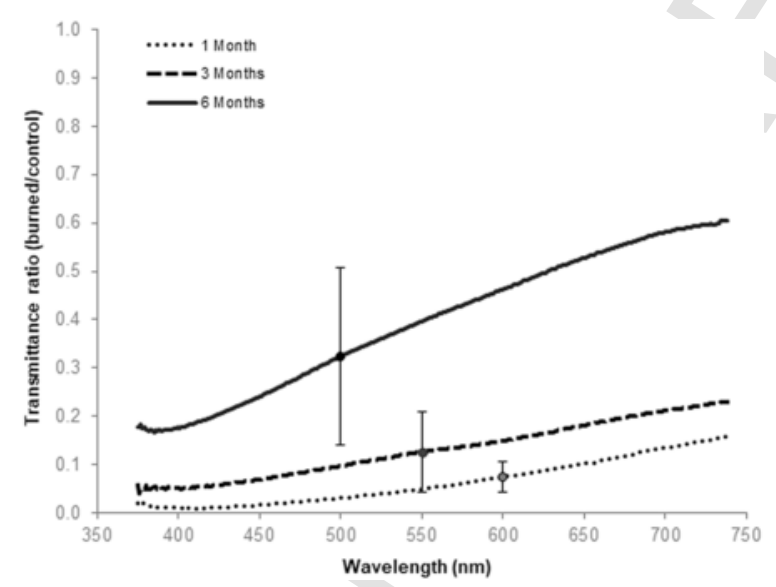

Fig. 2. Transmittance ratio (burned/control) of corneas at 1, 3, and 6 months after burning. Transmittance improved during the follow-up period but did not reach control levels.
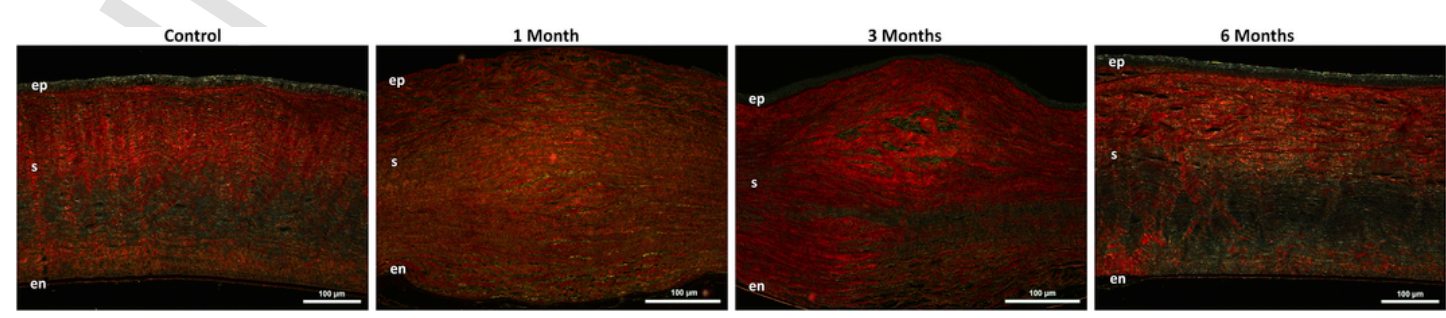

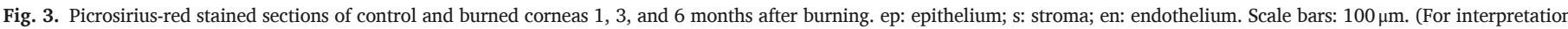
of the references to color in this figure legend, the reader is referred to the Web version of this article.) 


\section{Control}
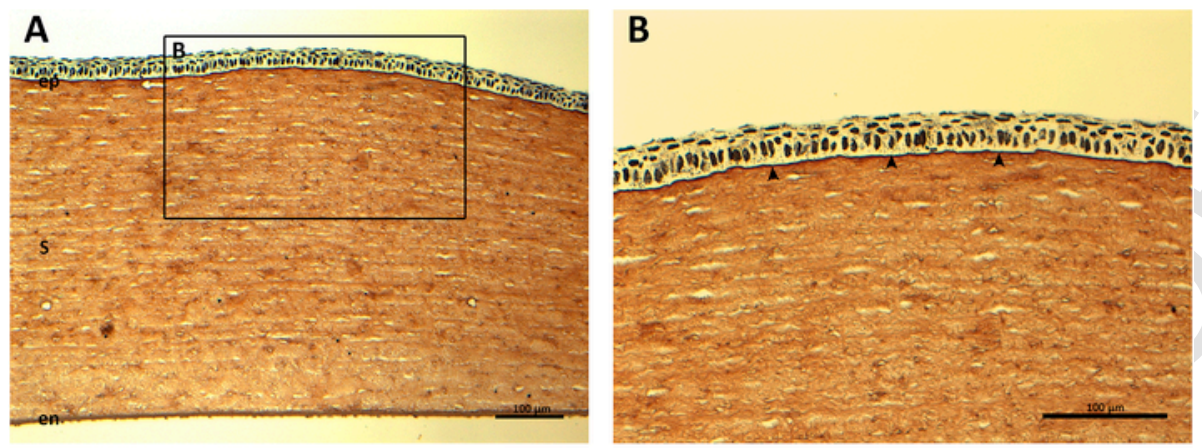

\section{Month}
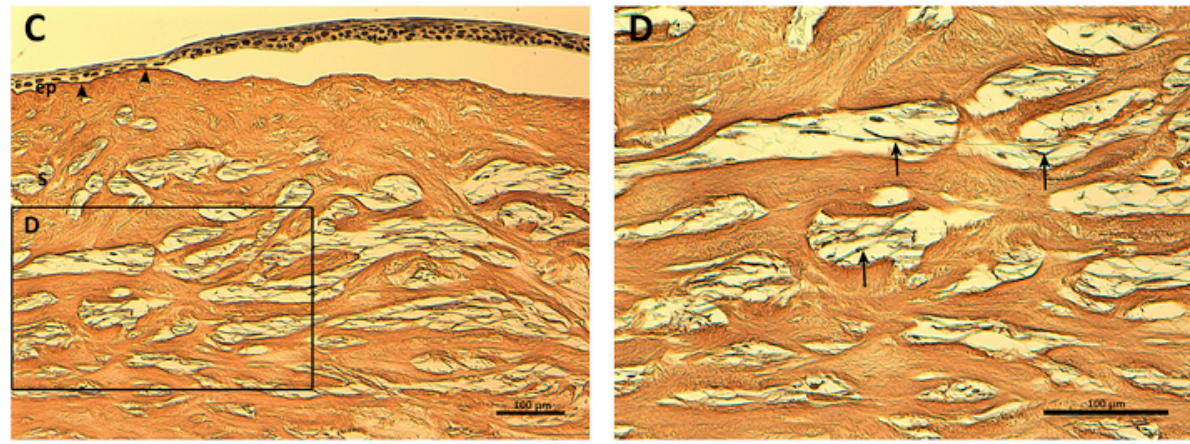

3 Months

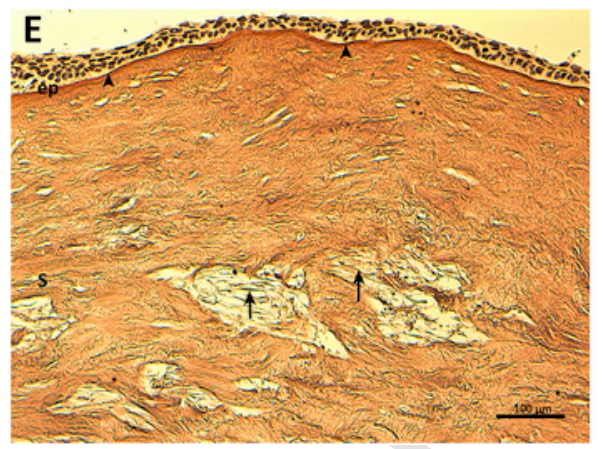

6 Months

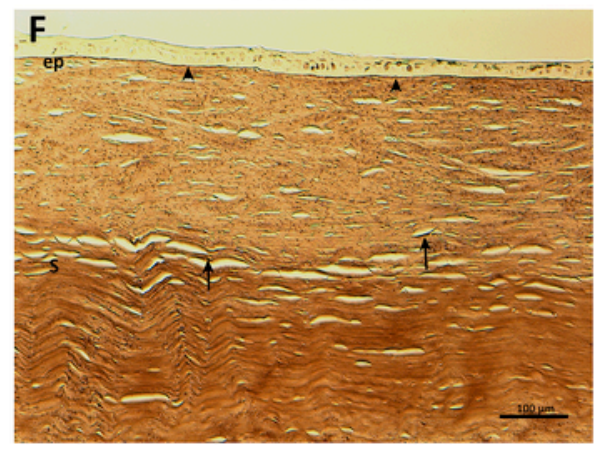

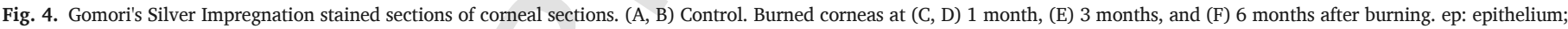
s: stroma; en: endothelium. Basement membrane (arrowheads) and collagen type III fibers (arrows) were stained black. (B) detail of (A) and (D) detail of (C). Scale bars: $100 \mu \mathrm{m}$.

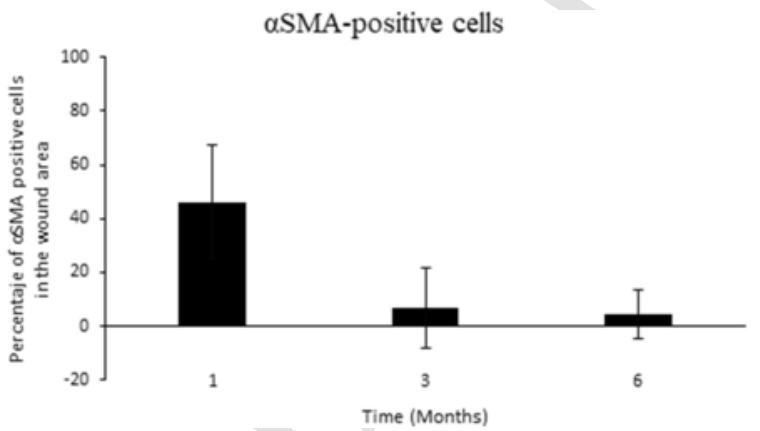

Fig. 5. Percentage of $\alpha$ SMA-positive cells (myofibroblasts) located in the wound area at the different study times. There were no significant differences although differences between 1 month and the other time points were near to the significance level.

\subsubsection{Transmission electron microscopy}

TEM images of control corneas showed the presence of keratocytes orientated parallel to the epithelium (Fig. 6A) and located between the lamellae collagen fibers that were orthogonally from each other (Fig. 6B and C). One month after burning, lacunae were filled with collagen fibers, and a variety of cells, mainly myofibroblasts (Fig. 6D). Myofibroblasts could be identify by their large cytoplasm with a lot of organelles, extensive endoplasmic reticulum and nucleus with euchromatin indicating transcriptional activity (Fig. 6E, *). There were other cells in the lacunae with fusiform cytoplasm, less organelles and condensate chromatin that indicate a great differentiation grade (Fig, 6E, ${ }^{* *}$ ). Moreover, at this time point, collagen fibers showed disorganization and gaps between fibers (Fig. 6F). Three months after injury, lacunae were present in the anterior stroma with more extensive cells (Fig. 6G and H) and collagen fibers started to show a clear orientation in lamellae but there were still some gaps and areas without clear organization (Fig. 6I). At the end of the study, there were not lacunae, cells were orientated once again parallel to surface (Fig. $6 \mathrm{~J}$ and $\mathrm{K}$ ) and collagen fibers were almost normally organized (Fig. 6L).

\subsection{Relative mRNA expression levels}

The relative expression levels of COL1A1 mRNA were up-regulated in both the wound and peripheral areas 1 month after burning (Fig. 7A). The expression levels were similar to control levels after 3 months, but were 

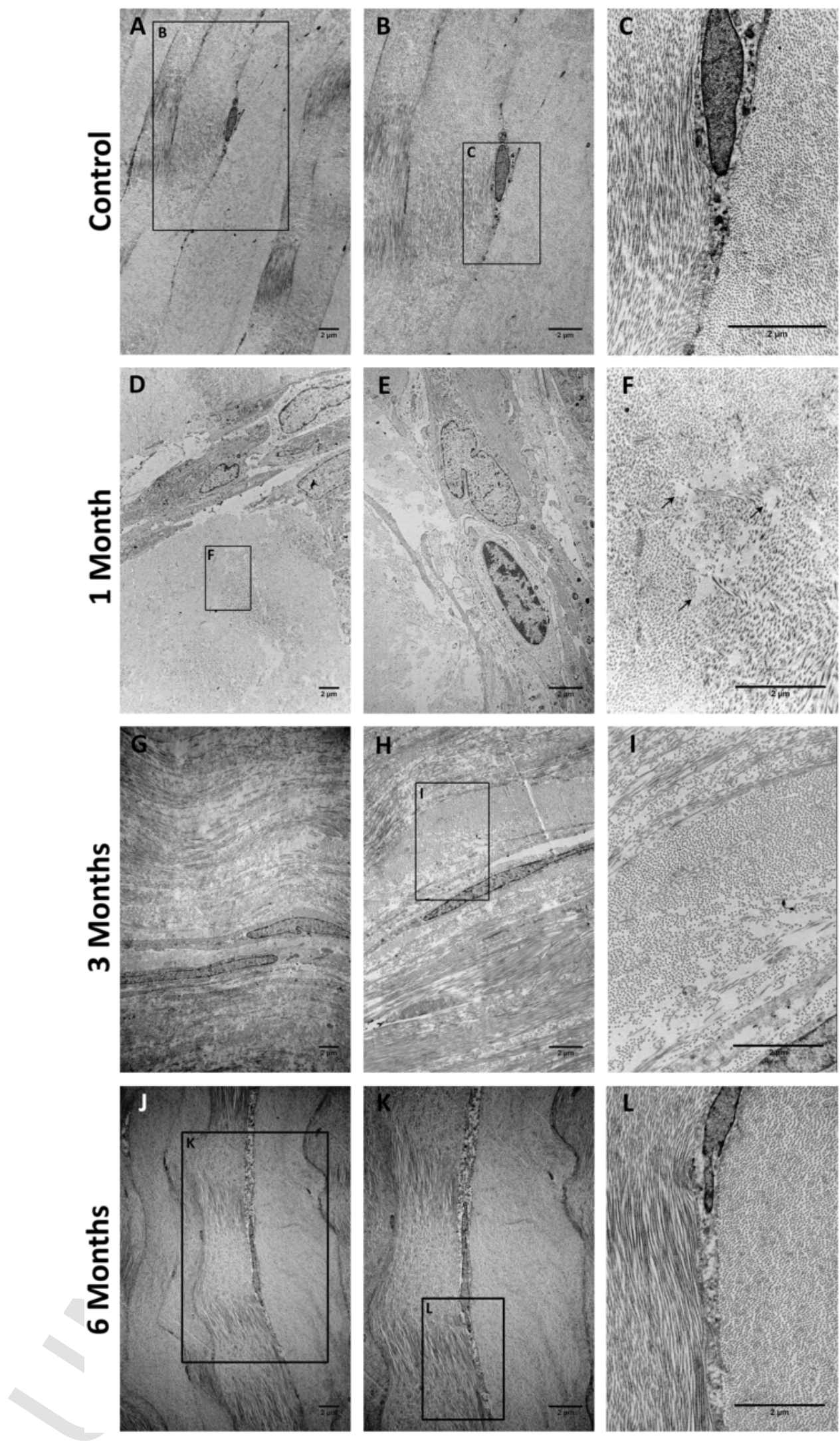

Fig. 6. Transmission electron microscopy (TEM) photographs. (A, B) Control cornea in which cells were orientated parallel to epithelium and collagen fibers formed lamellae. (C) Detail of (B), showing the orthogonally organization of collagen fiber lamellae. (D) Cornea 1 month after burning showing lacunae filled of several cell types. (E) Corneal lacunae 1 month after burning with a myofibroblast (*) and a cell with greater differentiation grade to keratocyte $\left.{ }^{(* *}\right)$. (F) Detail of (D), showing collagen fibers disorganization ang gaps between fibers (arrows). (G, H) Cornea 3 months after burning with more extensive cells. (I) Detail of (H), showing collagen fibers starting to organize in lamellae. (J, K) Cornea 6 months after burning without lacunae. (L) Detail of (K) with collagen fibers organized like control. Scale bars: $2 \mu \mathrm{m}$. 
A

COL1A1

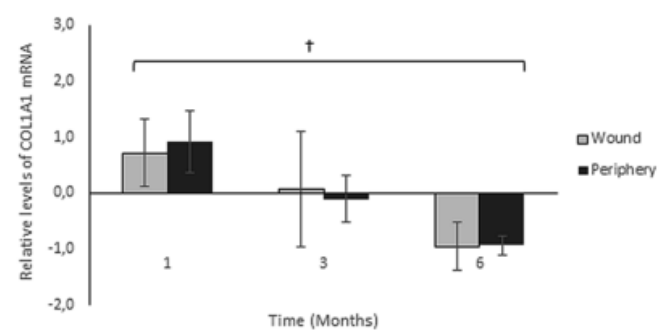

C

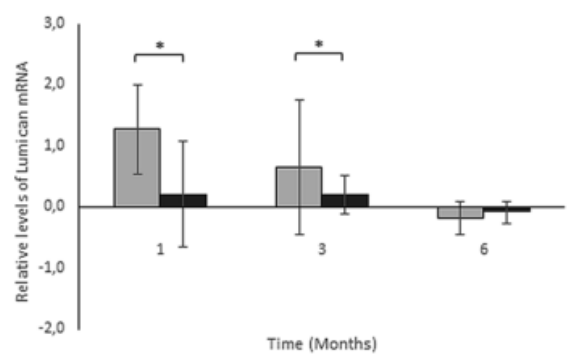

E

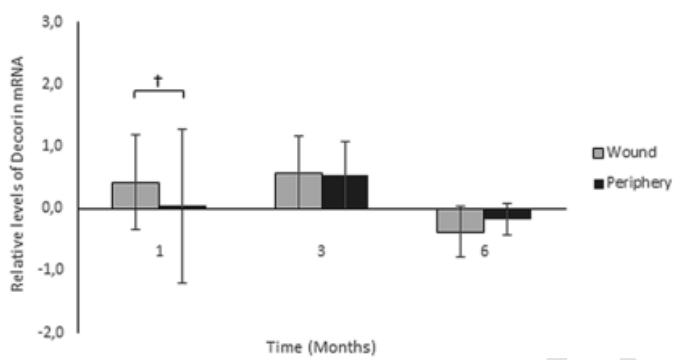

B

COL3A1

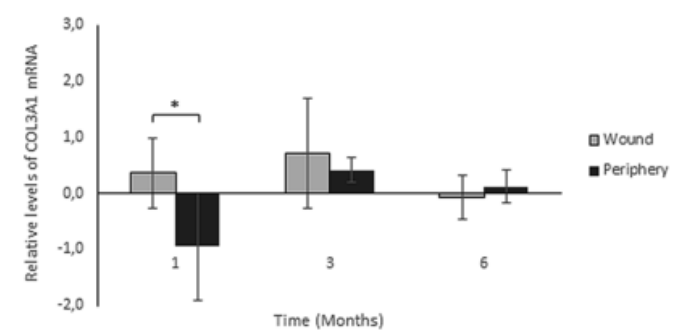

D

KERATOCAN

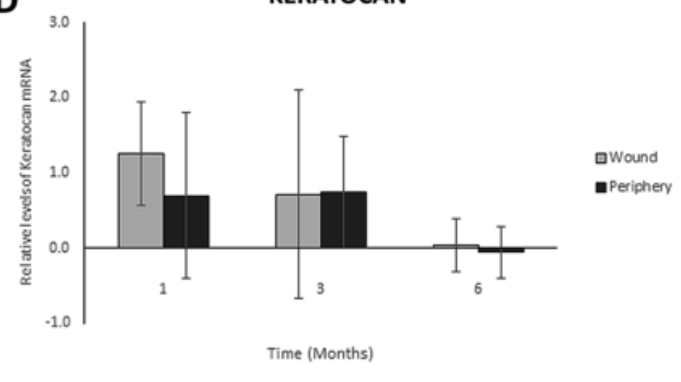

$\mathbf{F}$

$\alpha-S M A$

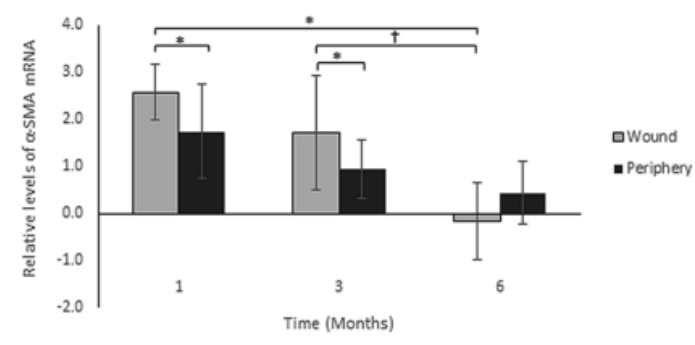

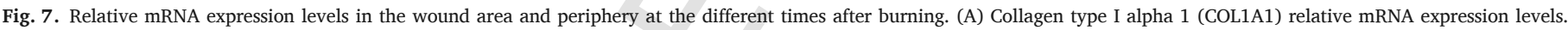

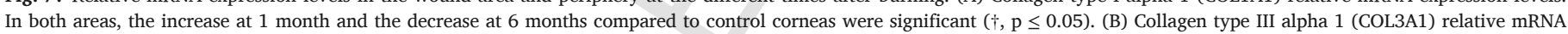

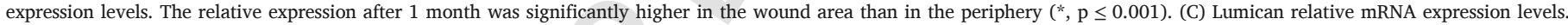

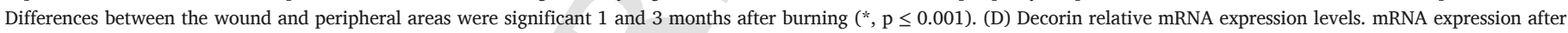

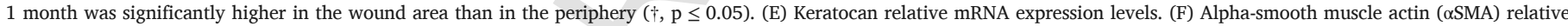

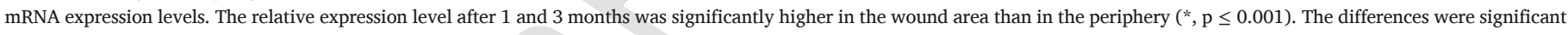
in wound area between 1 and 6 months $(*, p \leq 0.001)$, and 3 and 6 months (,$p \leq 0.05)$.

down-regulated at the end of the experiment. The differences between the wound and peripheral areas were not significant at any timepoint, but the differences between 1 and 6 months in both areas were significant ( $\mathrm{p} \leq 0.05$ for all comparisons).

One month after injury, COL3A1 mRNA expression levels were up-regulated in the wound area and down-regulated in periphery $(\mathrm{p} \leq 0.001$, Fig. 7B). The relative mRNA expression levels were up-regulated in both areas at 3 months after burning and normalized with regard to the control at 6 months. The differences between the two areas were not significant at either timepoint.

The dynamics of proteoglycan expression were similar to that observed for COL1A1. The relative mRNA expression levels of lumican and keratocan were up-regulated in the wound and peripheral areas at 1 and 3 months, and down-regulated at the end of the experiment (Fig. $7 C$ and D). Lumican mRNA expression levels were higher in the wound area than in the periphery at both 1 and 3 months $(\mathrm{p} \leq 0.001$ at both timepoints). Keratocan mRNA relative expression levels were higher in the wound area than in the periphery only 1 month after burning; there were no significant differences were found (Fig. 7D). The relative expression of decorin mRNA was up-regulated at 1 and 3 months after burning, and then down-regulated after 6 months in both the wound and peripheral areas (Fig. 7E). The difference between two areas was significant only at 1 month after burning ( $\mathrm{p} \leq 0.05)$.
The relative expression levels of $\alpha$ SMA mRNA were up-regulated in both the wound and peripheral areas at 1 and 3 months after burning, but only in periphery at 6 months (Fig. 7F). The differences between areas were significant at 1 and 3 months $(\mathrm{p} \leq 0.001)$. There were statistically significant differences in the wound area between 1 and 6 months, and 3 and 6 months $(\mathrm{p} \leq 0.05)$.

\section{Discussion}

Our study demonstrated the relationship between corneal opacity and changes in stromal collagen and proteoglycan expression after a severe alkali burn. Importantly, we monitored the recovery for six months, a relatively long period of time for this type of study, thus revealing the compositional changes within the stroma and how it becomes reorganized to recover transparency. Our multidisciplinary approach enabled a global understanding of the stromal wound healing process and can provide reliable and reproducible information that could allow the development of new and innovative treatments.

For descriptive purposes, the wound healing process after severe burns is usually divided into three phases (Huang et al., 2001). The acute phase begins within one week, during which cellular and structural damage remains in the wound area. The early reparative phase ensues and takes about 2-3 weeks, characterized by the presence of ulcer, edema, and increased cellular content. The late reparative phase takes place over 1-6 months during which corneal 
restoration occurs. A wide variety of corneal alkali burn models in rabbit have been described. Most studied the wound healing process and the effect of different treatments only in the acute and early reparative phases (Griffith GL et al., 2018; Rezaei Kanavi et al., 2016). Some studies included the late reparative phase (Huang et al., 2001; Kubota M, 1991), but they did not take a multidisciplinary approach in the examination of changes in the ECM. Thus, our study is the first to focus a multidisciplinary approach on ECM reorganization in the late reparative phase.

Due to the importance of collagen in normal and wounded corneas, it is necessary to characterize the compositional changes during the wound healing process. Because there are no reliable antibodies for immunodetection of rabbit collagen types I and III, we have utilized another technique. Picrosirius-red staining of the corneas, visualized under polarized light, allows the characterization of the collagen network because collagen is the major solid component that possesses anisotropy (Huang et al., 2001). However, there is controversy regarding the interpretation of the polarization colors. Some authors associated the colors with the collagen types (Bayounis et al., 2011; Binnebosel et al., 2010; Cavallo et al., 2014; Coen et al., 2013; Lattouf et al., 2014; Peeters et al., 2014), but others reported that the polarization colors depend only on the fiber thickness, spatial arrangement, and packing density (Coleman, 2011; Dayan et al., 1989). Lattoud et al. (Lattouf et al., 2014) demonstrated that phenotypic collagen determination by this method is unsuitable, and the polarization colors only reveal the collagen fiber organization and heterogeneity. To solve this concern and differentiate the collagen types, we stained the collagen type III fibers with the Gomori's silver impregnation (Gomori, 1937).

One month after injury, corneal opacity reached the highest values in the wounded area, producing loss of light transmittance, as was demonstrated previously (Huang et al., 2001). Picrosirius red and Gomori's silver impregnation stained sections showed a disorganized stroma with lacunae filled with thinner, less packed and misaligned fibers that correspond with collagen type III fibers. Our results agree with the lacunae description by Kubota (Kubota, 1991) and with the presence of a disorganized ECM synthetized by myofibroblasts localized in the anterior and middle stroma as described by Gallego-Munoz et al. and Huang et al. (Gallego-Munoz et al., 2019; Huang et al., 2001) and demonstrated by the percentage of $\alpha$ SMA-positive cells at this time point in the present study. TEM images showed lacunae with cells in several differentiation states and disorganized collagen fibers.

We observed a significant up-regulation of collagen type I and III mRNA expression levels in the wound area. This was consistent with previous in vitro studies that also found an up-regulation of collagens type I and III by cultured human myofibroblasts and cultured bovine myofibroblasts (Funderburgh et al., 2001; Gallego-Munoz et al., 2018). The presence of both collagen types has been described during corneal scarring by Massoudi et al. (2016), indicating the presence of a disorganized ECM that was related to the loss of transparency, as we found here. Simultaneously, relative mRNA expression levels of the proteoglycans lumican, keratocan and decorin, necessary for the regular assembly of collagen during wound healing, were upregulated in the wound area. The inactivation of lumican or decorin genes in mice produces irregular collagen ultrastructure with development of corneal opacity (Neame et al., 2000). Keratocan-deficient mice show larger diameters of corneal collagen fibers but less organized packing and reduced thickness of corneal stroma (Liu et al., 2003). This demonstrate the importance of these proteoglycans in collagen fibril formation in the maintenance and restoration of corneal transparency.

Three months after corneal wounding, there was a reduction in corneal opacity, a decrease of myofibroblasts percentage and an increase in transmittance ratio of burned to control corneas. While there were fewer stromal lacunae at that time, they were larger, and the collagen type III fibers remained in the wound area. TEM images showed that collagen fiber organization improved. Collagen type III and proteoglycan expression levels were still up-regulated, indicating the need to synthesize more of these molecules to restore the stromal organization and transparency. Collagen type I mRNA expression was similar to that of normal corneas, indicating wound healing progress.

At the end of the six-month follow-up period, corneal transparency necessity for new synthesis of ECM components. Light transmittance through the corneas was improved, and like control corneas, it was dependent on wavelength (Farrell et al., 1973). However, the transmittance values remained lower than control corneas, indicating that the stromal structure was not fully restored. Our device only measured direct transmissivity, which is related to an increase in scattered light (Mar et al., 2009) that we did not directly detect. The high level of scattering that remained in the severely injured corneas is probably due to the absence of the normal stromal architecture, which was partially replaced by the cells that appear in the healing process (Mar et al., 2009) and the lacunae or voids that appear in the stroma (McCally et al., 2007). Direct measurement of light scattering during the wound healing process could support our transmittance data and give more information about stromal restoration after an alkali burn.

In summary, we developed a deeper understanding of the time-dependent stages of wound recovery from a severe alkali burn. Over the six months of study, we followed the restructuring of the collagen organization and the ECM composition. With this new knowledge, we can begin to design anti-scarring therapies and test the effectiveness of existing and newly developed drugs.

\section{Funding}

This work was supported by PI15/01906 Health Research Fund from the Instituto de Salud Carlos III. Ministerio de Economía y Competitividad, Spain. P. Cidad was supported with the grants from Ministerio de Economía Competitividad BFU2016-75360-R and Junta de Castilla y León VA114P17.

\section{Acknowledgments}

We thank to Cristina Herrero-Pérez for her technical assistance, Luis Santiago and Manuel Avella for their transmission electronic technical assistance and Britt Bromberg, PhD, ELS, of Xenofile Editing (www. xenofileediting.com) for the editing of the manuscript.

\section{References}

Barbosa, F.L., Chaurasia, S.S., Cutler, A., Asosingh, K., Kaur, H., de Medeiros, F.W., Agrawal, V., Wilson, S.E., 2010. Corneal myofibroblast generation from bone marrow-derived cells. Exp. Eye Res. 91, 92-96.

Bayounis, A.M., Alzoman, H.A., Jansen, J.A., Babay, N., 2011. Healing of peri-implant tissues after flapless and flapped implant installation. J. Clin. Periodontol. 38, 754-761.

Binnebosel, M., Klink, C.D., Otto, J., Conze, J., Jansen, P.L., Anurov, M., Schumpelick, V., Junge, K., 2010. Impact of mesh positioning on foreign body reaction and collagenous ingrowth in a rabbit model of open incisional hernia repair. Hernia : J. Hernias Abdom. Wall Surg. 14, 71-77.

Boote, C., Dennis, S., Newton, R.H., Puri, H., Meek, K.M., 2003. Collagen fibrils appear more closely packed in the prepupillary cornea: optical and biomechanical implications. Investig. Ophthalmol. Vis. Sci. 44, 2941-2948.

Cavallo, J.A., Roma, A.A., Jasielec, M.S., Ousley, J., Creamer, J., Pichert, M.D., Baalman, S., Frisella, M.M., Matthews, B.D., Deeken, C.R., 2014. Remodeling characteristics and collagen distribution in synthetic mesh materials explanted from human subjects after abdominal wall reconstruction: an analysis of remodeling characteristics by patient risk factors and surgical site classifications. Surg. Endosc. 28, 1852-1865.

Chakravarti, S., 2006. Focus on molecules: keratocan (KERA). Exp. Eye Res. 82, 183-184.

Chaurasia, S.S., Lim, R.R., Lakshminarayanan, R., Mohan, R.R., 2015. Nanomedicine approaches for corneal diseases. J. Funct. Biomater. 6, 277-298.

Chen, L., Kato, T., Toshida, H., Nakamura, S., Murakami, A., 2005. Immunohistochemical characterization of epithelial cells implanted in the flap-stroma interface of the cornea. Jpn. J. Ophthalmol. 49, 79-83.

Chen, S., Mienaltowski, M.J., Birk, D.E., 2015. Regulation of corneal stroma extracellular matrix assembly. Exp. Eye Res. 133, 69-80.

Choi, H., Phillips, C., Oh, J.Y., Stock, E.M., Kim, D.K., Won, J.K., Fulcher, S., 2017. Comprehensive modeling of corneal alkali injury in the rat eye. Curr. Eye Res. 42 , 1348-1357.

Chung, J.H., Fagerholm, P., Lindstrom, B., 1987. The behaviour of corneal epithelium following a standardized alkali wound. Acta Ophthalmol. 65, 529-537.

Coen, M., Menegatti, E., Salvi, F., Mascoli, F., Zamboni, P., Gabbiani, G., Bochaton-Piallat, M.L., 2013. Altered collagen expression in jugular veins in multiple sclerosis. Cardiovasc. Pathol. : Off. J. Soc. Cardiovasc. Pathol. 22, 33-38.

Coleman, R., 2011. Picrosirius red staining revisited. Acta Histochem. 113, 231-233.

Dayan, D., Hiss, Y., Hirshberg, A., Bubis, J.J., Wolman, M., 1989. Are the polarization colors of picrosirius red-stained collagen determined only by the diameter of the fibers? Histochemistry $93,27-29$. 
Farrell, R.A., McCally, R.L., Tatham, P.E., 1973. Wave-length dependencies of light scattering in normal and cold swollen rabbit corneas and their structural implications. J. Physiol. 233, 589-612.

Funderburgh, J.L., Funderburgh, M.L., Mann, M.M., Corpuz, L., Roth, M.R., 2001. Proteoglycan expression during transforming growth factor beta -induced keratocyte-myofibroblast transdifferentiation. J. Biol. Chem. 276, 44173-44178.

Gabbiani, G., 2003. The myofibroblast in wound healing and fibrocontractive diseases. J. Pathol. 200, 500-503.

Galiacy, S.D., Fournie, P., Massoudi, D., Ancele, E., Quintyn, J.C., Erraud, A., Raymond-Letron, I., Rolling, F., Malecaze, F., 2011. Matrix metalloproteinase 14 overexpression reduces corneal scarring. Gene Ther. 18, 462-468.

Gallego-Munoz, P., Ibares-Frias, L., Garrote, J.A., Valsero-Blanco, M.C., Cantalapiedra-Rodriguez, R., Merayo-Lloves, J., Carmen Martinez-Garcia, M., 2018. Human corneal fibroblast migration and extracellular matrix synthesis during stromal repair: role played by platelet-derived growth factor-BB, basic fibroblast growth factor, and transforming growth factor-beta1. J. Tissue Eng. Regenerat. Med. 12, e737-e746.

Gallego-Munoz, P., Lorenzo-Martin, E., Fernandez, I., Herrero-Perez, C., Martinez-Garcia, M.C., 2019. Nidogen-2: location and expression during corneal wound healing. Exp. Eye Res. 178, 1-9.

Gomori, G., 1937. Silver impregnation of reticulum in paraffin sections. Am. J. Pathol. 13, 993-1002, 1005.

Griffith GL, W.B., Lee, H.K., Cornell, L.E., McDaniel, J.S., Zamora, D.O., Johnson, A.J., 2018. Treatment of corneal chemical alkali burns with a crosslinked thiolated hyaluronic acid film. Burns 44, 1179-1186.

Gupta, N., Kalaivani, M., Tandon, R., 2011. Comparison of prognostic value of Roper Hall and Dua classification systems in acute ocular burns. Br. J. Ophthalmol. 95, 194-198.

Haddox, J.L., Pfister, R.R., Sommers, C.I., Blalock, J.E., Villain, M., 2001. Inhibitory effect of a complementary peptide on ulceration in the alkali-injured rabbit cornea. Investig. Ophthalmol. Vis. Sci. 42, 2769-2775.

Hassell, J.R., Birk, D.E., 2010. The molecular basis of corneal transparency. Exp. Eye Res. 91, 326-335.

Hatami-Marbini, H., Etebu, E., 2013. Hydration dependent biomechanical properties of the corneal stroma. Exp. Eye Res. 116, 47-54.

Huang, Y., Meek, K.M., Ho, M.W., Paterson, C.A., 2001. Analysis of birefringence during wound healing and remodeling following alkali burns in rabbit cornea. Exp. Eye Res. 73, 521-532.

Junqueira, L.C., Bignolas, G., Brentani, R.R., 1979. Picrosirius staining plus polarization microscopy, a specific method for collagen detection in tissue sections. Histochem. J. $11,447-455$.

Karsdal, M.A., Nielsen, S.H., Leeming, D.J., Langholm, L.L., Nielsen, M.J., Manon-Jensen, T., Siebuhr, A., Gudmann, N.S., Ronnow, S., Sand, J.M., Daniels, S.J., Mortensen, J.H., Schuppan, D., 2017. The good and the bad collagens of fibrosis - their role in signaling and organ function. Adv. Drug Deliv. Rev. 121, 43-56.

Kaur, H., Chaurasia, S.S., Agrawal, V., Suto, C., Wilson, S.E., 2009. Corneal myofibroblast viability: opposing effects of IL-1 and TGF beta1. Exp. Eye Res. 89, 152-158.

Klingberg, F., Hinz, B., White, E.S., 2013. The myofibroblast matrix: implications for tissue repair and fibrosis. J. Pathol. 229, 298-309.

Kubota, M.,F.P., 1991. Corneal alkali burn in the rabbit. Acta Ophtalmol. 69, 635-340.

Lattouf, R., Younes, R., Lutomski, D., Naaman, N., Godeau, G., Senni, K., Changotade, S., 2014. Picrosirius red staining: a useful tool to appraise collagen networks in normal and pathological tissues. J. Histochem. Cytochem. : Off. J. Histochem. Soc.y 62, 751-758.

Leask, A., Abraham, D.J., 2004. TGF-beta signaling and the fibrotic response. FASEB J. 18, 816-827.
Liu, C.Y., Birk, D.E., Hassell, J.R., Kane, B., Kao, W.W., 2003. Keratocan-deficient mice display alterations in corneal structure. J. Biol. Chem. 278, 21672-21677.

Lopes, G.J.A., Casella, A.M.B., Oguido, A.P., Matsuo, T., 2017. Effects of topical and subconjunctival use of bevacizumab on corneal neovascularization in rabbits' eyes. Arq Bras. Oftalmol. 80, 252-256.

Mar, S., Martinez-Garcia, M.C., T, B.-M., RM, T., Merayo-Lloves, J., 2009. Measurement of correlation between transmission and scattering during wound healing in hen corneas. J. Mod. Opt. 56, 1-8.

Massoudi, D., Malecaze, F., Galiacy, S.D., 2016. Collagens and proteoglycans of the cornea: importance in transparency and visual disorders. Cell Tissue Res. 363, 337-349.

Maurice, D.M., 1970. The transparency of the corneal stroma. Vis. Res. 10, 107-108.

McCally, R.L., Freund, D.E., Zorn, A., Bonney-Ray, J., Grebe, R., de la Cruz, Z., Green, W.R., 2007. Light-scattering and ultrastructure of healed penetrating corneal wounds. Investig. Ophthalmol. Vis. Sci. 48, 157-165.

Michelacci, Y.M., 2003. Collagens and proteoglycans of the corneal extracellular matrix. Brazi. J. Med. Biol. Res. = Revista brasileira de pesquisas medicas e biologicas 36, 1037-1046.

Neame, P.J., Kay, C.J., McQuillan, D.J., Beales, M.P., Hassell, J.R., 2000. Independent modulation of collagen fibrillogenesis by decorin and lumican. Cell. Mol. Life Sci. CMLS 57, 859-863.

Peeters, E., De Hertogh, G., Junge, K., Klinge, U., Miserez, M., 2014. Skin as marker for collagen type I/III ratio in abdominal wall fascia. Hernia : J. Hernias Abdom. Wall Surg. 18, 519-525.

Petroll, W.M., Miron-Mendoza, M., 2015. Mechanical interactions and crosstalk between corneal keratocytes and the extracellular matrix. Exp. Eye Res. 133, 49-57.

Radisky, D.C., Kenny, P.A., Bissell, M.J., 2007. Fibrosis and cancer: do myofibroblasts come also from epithelial cells via EMT?. J. Cell. Biochem. 101, 830-839.

Radner, W., Zehetmayer, M., Aufreiter, R., Mallinger, R., 1998. Interlacing and cross-angle distribution of collagen lamellae in the human cornea. Cornea 17, 537-543.

Rezaei Kanavi, M., Tabeie, F., Sahebjam, F., Poursani, N., Jahanbakhsh, N., Paymanpour, P., AfsarAski, S., 2016. Short-term effects of extremely low-frequency pulsed electromagnetic field and pulsed low-level laser therapy on rabbit model of corneal alkali burn. Exp. Eye Res. 145, 216-223.

Ricard-Blum, S., 2011. The collagen family. Cold Spring Harb. Perspect Biol. 3, a004978.

Saika, S., Yamanaka, O., Sumioka, T., Miyamoto, T., Miyazaki, K., Okada, Y., Kitano, A., Shirai, K., Tanaka, S., Ikeda, K., 2008. Fibrotic disorders in the eye: targets of gene therapy. Prog. Retin. Eye Res. 27, 177-196.

Scott, J.E., Haigh, M., 1985. 'Small'-proteoglycan:collagen interactions: keratan sulphate proteoglycan associates with rabbit corneal collagen fibrils at the 'a' and 'c' bands. Biosci. Rep. 5, 765-774.

Sharma, N., Kaur, M., Agarwal, T., Sangwan, V.S., Vajpayee, R.B., 2018. Treatment of acute ocular chemical burns. Surv. Ophthalmol. 63, 214-235.

Singh, V., Agrawal, V., Santhiago, M.R., Wilson, S.E., 2012. Stromal fibroblast-bone marrow-derived cell interactions: implications for myofibroblast development in the cornea. Exp. Eye Res. 98, 1-8.

Tandon, A., Tovey, J.C., Sharma, A., Gupta, R., Mohan, R.R., 2010. Role of transforming growth factor Beta in corneal function, biology and pathology. Curr. Mol. Med. 10, 565-578.

West-Mays, J.A., Dwivedi, D.J., 2006. The keratocyte: corneal stromal cell with variable repair phenotypes. Int. J. Biochem. Cell Biol. 38, 1625-1631.

Zahir-Jouzdani, F.,S.M., Mahbod, M., Mottaghitalab, F., Vakhsite, F., Arefian, E., Shahhoseini, S., Dinarvand, R., Atyabi, F., 2018. Corneal chemical burn treatment through a delivery system consisting of TGF-B1 siRNA: in vitro and in vivo. Drug Deliv. Transl. Res. 8, 1127-1138.

Zhang, K., Rekhter, M.D., Gordon, D., Phan, S.H., 1994. Myofibroblasts and their role in lung collagen gene expression during pulmonary fibrosis. A combined immunohistochemical and in situ hybridization study. Am. J. Pathol. 145, 114-125. 